\title{
A FIRST ORDER PROJECTION-BASED TIME-SPLITTING SCHEME FOR COMPUTING CHEMICALLY REACTING FLOWS
}

\author{
ANDREAS PROHL ${ }^{1}$
}

\begin{abstract}
The simulation of chemically reacting flows in specific situations is a basic instrument in natural sciences in order to understand complex phenomena (e.g., salt concentrations in oceans) as well as in engineering sciences (e.g., the optimization of the Czochralski growth in semiconductor industries, see e.g. $[7,15])$. The objective of the paper is two-fold: First, we will present a first-order time-splitting scheme that is suitable for parallelization of the related quantities in each time-step. Additionally, this scheme is based on the separate computation of the new velocity-field and pressure by means of Chorin's scheme. Second, we present a thorough analysis of this scheme deriving optimal error statements that are applicable for general flow situations.
\end{abstract}

Key words. Chemically reacting flows, Boussinesq model, incompressible Navier-Stokes equations, convergence analysis, projection methods, splitting methods, Chorin scheme.

AMS subject classifications. 35Q35, 65M12, 76D05, 76V05.

1 Introduction. The modeling of chemically reacting flows is important for the analysis and control of manufacturing processes, surface catalytic reactors for methane to methanol conversion and chemical vapor deposition (CVD) process modeling for production of advanced semiconductor materials. As far as the last application is concerned, designers of CVD reactors need detailed information on the complex flow structure, temperature distribution, chemical species distribution and the uniformity of deposition rates. In a typical reaction mechanism, there can be over thirty important species undergoing more than fifty reactions, see $[1,7,10,14]$.

The goal of the work is to propose a time-splitting scheme for the system of equations that describe the dynamics of the chemically reacting flow. They are derived from the conservation principles for impulse, mass and energy. If we consider a bounded domain $\Omega \subset \mathrm{R}^{d}, d=2,3$ where the reactive flow is in and observe its behavior over a period of time $\left[0, t_{M+1}\right]$, the dynamics is described by the system of equations

$$
\begin{aligned}
& u_{t}-\operatorname{Pr} \Delta u+(u \cdot \nabla) u+\nabla p=f_{0}(T), \\
& \operatorname{div} u=0 \\
& T_{t}-\Delta T+(u \cdot \nabla) T=-\sum_{i=1}^{N} h_{i} W_{i}\left(\left\{Y_{i}\right\}_{i=1}^{N}, T\right), \\
& Y_{i, t}-\frac{1}{L e} \Delta Y_{i}+(u \cdot \nabla) Y_{i}=W_{i}\left(\left\{Y_{i}\right\}_{i=1}^{N}, T\right), \quad i=1, \ldots, N .
\end{aligned}
$$

\footnotetext{
${ }^{1}$ Mathematisches Seminar, Christian-Albrechts-Universität Kiel, Ludewig-Meyn-Str. 4, D-24098 Kiel, Germany (apr@numerik.uni-kiel.de).
} 
For simplicity, we suppose the following initial and boundary value data for the problem under consideration,

$$
\begin{aligned}
& \left.u\right|_{\partial \Omega}=0,\left.\quad T\right|_{\partial \Omega}=0,\left.\quad Y_{i}\right|_{\partial \Omega}=0, \\
& u(0)=u_{0} \in \mathbf{J}_{1} \cap \mathbf{H}^{2}, \quad T(0)=T_{0} \in H_{0}^{1} \cap H^{2}, \quad Y_{i}(0)=Y_{i}^{0} \in H_{0}^{1} \cap H^{2} .
\end{aligned}
$$

In the presented model, $\mathrm{Pr}$ is the Prandl number, Le the Lewis number that scale the different diffusive and the convective characters in the equations. In the following studies, they will be set equal to $1 . p=p(x, t)$ is the scalar pressure and $u=u(x, t) \in \mathrm{R}^{d}$ the solenoidal velocity field. The flow is driven by the buoyant forcing term $f_{0}(T)$ as a function of the temperature $T=T(x, t)$, by making use of the Boussinesq model. Finally, the $N$ tuple $\left\{Y_{i}\right\}_{i=1}^{N}$ determines the mass fractions $Y_{i}=Y_{i}(x, t)$ of the species indexed by $i$ of the chemically reacting fluid flow in space and time. Owing to the mass conservation principle, they satisfy

$$
0 \leq Y_{i} \leq 1, \quad \text { and } \quad \sum_{i=1}^{N} Y_{i}=1, \quad \sum_{i=1}^{N} W_{i}=0, \quad \forall(x, t) \in \Omega \times\left[0, t_{M+1}\right] .
$$

The value $h_{i}$ is the enthalpy of the species $i$ divided by its molecular weight, i.e., a measure of the amount of heat that is contained in species $i$.

The chemical reactions between the diverse species are described by means of the Arrhenius model in which the net production/removal rates $W_{i}$ take the form

$$
W_{i}\left(\left\{Y_{i}\right\}_{i=1}^{N}, T\right)=\sum_{j=1}^{m_{i}} A_{j} e^{-E_{j} / R_{0} T} \prod_{k=1}^{N} C_{j}^{\nu_{j, k}},
$$

with $A_{j}$ the frequency factors, $E_{j}$ the activation energies, $R_{0}$ the universal gas constant, and $C_{i}$ the concentrations, i.e., the mass fraction $Y_{i}$ divided by the molecular weight. The $\nu_{j, k}$ are nonegative integers, where at least one of the $\nu_{j, k}$ for $k=1, \ldots, N$ is nonzero, for each $j$.

In the subsequent analysis, we abstract from the specific form of the change of mass fractions, and make the following assumptions:

1. The $W_{i}$ are Lipschitz functions, $W_{i} \in C^{0,1}\left(0, T ; \prod_{i=1}^{N+1} L^{2}\right)$. In particular, each $W_{i}$ is bounded,

$$
\left|W_{i}\left(\left\{Y_{i}\right\}_{i=1}^{N}, T\right)\right| \leq C, \quad \forall\left(\left\{Y_{i}\right\}_{i=1}^{N}, T\right) \in[0,1]^{N} \times[0, \infty), \quad 1 \leq i \leq N .
$$

2. The mass conservation implies the relation

$$
\sum_{i=1}^{N} W_{i}\left(\left\{Y_{i}\right\}_{i=1}^{N}, T\right)=0, \quad \forall\left(\left\{Y_{i}\right\}_{i=1}^{N}, T\right) \in[0,1]^{N} \times[0, \infty) .
$$

The well-posedness of (1), (2) has been shown in [9, 16], and the analysis has been extended in [12] to practically more relevant boundary conditions. For further discussions, especially on the chemical background, we refer to $[11,12]$. 
2 Presentation of the time-splitting scheme. The effective time-discretization of system (1) is made difficult through a couple of problems. First, the coupling of the diverse functions in the 'flow part' and the 'chemical part' gives rise to large discretization matrices that limit the flexibility of a fully implicit discretization approach, due to limited computational resources. Second, we have to choose a stable finite element discretization pairing for the velocity and pressure fields to satisfy the $L B B$-condition of Ladyszhenskaya, Babuska and Brezzi, cf. [2]. Finally, the nonlinear parts given by the reaction terms and the convective term in the first equation necessitate a careful numerical treatment.

In order to significantly reduce the computational effort, we propose a splitting scheme that decouples the computation of velocity and pressure, temperature and the $N$ mass fraction functions in each iteration step. The stiffness matrices for the computation of velocity field, temperature and mass fractions that arise from a spatial discretization will then be generated from the knowledge of the previous velocity field, temperature and mass fractions and the solution process itself can then be done in parallel. Furthermore, the decoupling of the computation of velocity field and pressure iterate is accomplished through the classical projection method of Chorin, see $[3,4]$. The scheme then reads:

Given $\left\{u^{m}, T^{m},\left\{Y_{i}^{m}\right\}_{i=1}^{N}\right\}$, determine $\left\{u^{m+1}, p^{m+1}, T^{m+1},\left\{Y_{i}^{m+1}\right\}_{i=1}^{N}\right\} \in\left(\mathbf{J}_{\mathbf{0}} \cap \mathbf{H}^{2}\right) \times$ $H^{1} / \mathrm{R} \times\left(H_{0}^{1} \cap H^{2}\right) \times \prod_{i=1}^{N}\left(H_{0}^{1} \cap H^{2}\right)$ in the following way.

1. Start with an initial guess $u^{0}=u_{0}, T^{0}=T_{0},\left\{Y_{i}^{0}\right\}_{i=1}^{N}=\left\{Y_{i}(0)\right\}_{i=1}^{N}$. Then, for $m \geq 0$, the following steps determine the iterates.

2. Find $\tilde{u}^{m+1}$ that solves

$$
\frac{1}{k}\left\{\tilde{u}^{m+1}-u^{m}\right\}-\Delta \tilde{u}^{m+1}+\left(u^{m} \cdot \nabla\right) \tilde{u}^{m+1}=f_{0}\left(T^{m}\right),
$$

3. Determine the tuple $\left\{u^{m+1}, p^{m+1}\right\}$ that solves the system

$$
\begin{aligned}
& \frac{1}{k}\left\{u^{m+1}-\tilde{u}^{m+1}\right\}+\nabla p^{m+1}=0 \\
& \operatorname{div} u^{m+1}=0,\left.\quad u^{m+1}\right|_{\partial \Omega} \cdot n=0
\end{aligned}
$$

4. Compute $T^{m+1}$ that is the solution of

$$
\frac{1}{k}\left\{T^{m+1}-T^{m}\right\}-\Delta T^{m+1}+\left(u^{m} \cdot \nabla\right) T^{m+1}=-\sum_{i=1}^{N} h_{i} W_{i}\left(\left\{Y_{i}^{m}\right\}_{i=1}^{N}, T^{m+1}\right),
$$

5. The $N$-tuple $\left\{Y_{i}^{m+1}\right\}_{i=1}^{N}$ is governed by

$$
\frac{1}{k}\left\{Y_{i}^{m+1}-Y_{i}^{m}\right\}-\Delta Y_{i}^{m+1}+\left(u^{m} \cdot \nabla\right) Y_{i}^{m+1}=W_{i}\left(\left\{Y_{i}^{m+1}\right\}_{i=1}^{N}, T^{m}\right),
$$


with $k>0$ being the time-step used in the scheme. - Note that the computation of the diverse functions at each time-step can be accomplished in a fully decoupled manner. Furthermore, we can benefit from this approach by parallelizing the computation in each iteration step. In this respect, we recall that (5) can be reformulated in the following way. Applying the div operator amounts to solving a Laplace-Neumann problem for the pressure iterate,

$$
-\Delta p^{m+1}=-\frac{1}{k} \operatorname{div} \tilde{u}^{m+1},\left.\quad \partial_{n} p^{m+1}\right|_{\partial \Omega}=0
$$

followed by an algebraic update for the solenoidal velocity field,

$$
u^{m+1}=\tilde{u}^{m+1}-k \nabla p^{m+1} .
$$

This decoupling strategy in the computation of the velocity field and pressure iterate has been proposed by Chorin and is known as a first order projection scheme for solving the incompressible Navier-Stokes equations, see [3, 4].

The goal of the remainder of the present paper is devoted to an analysis of the scheme (4) through (7) by investigating its stability and approximation properties. There are distinct error mechanisms acting in the present scheme, that are subject to the subsequent investigation. We have to quantify the effects of the projection scheme approach as well as those of the decoupling of the equations in (1). In doing so, we propose a series of auxiliary problems in section 4 that are devoted to the study of these distinct error mechanisms inherent to (4) through (7). We mention, that the subsequent study heavily relies on results that have been obtained for Chorin's projection scheme in [13]. A brief summary of results for Chorin's projection method that are relevant for the present analysis is given in section 4 .

For the subsequent analysis, we need some technical assumptions regarding the regularity of the given problem data. In the following, we often refer to the spaces

$$
\mathbf{J}_{0}=\left\{v \in \mathbf{L}^{2}, \operatorname{div} v=0 \text { and }\left.v\right|_{\partial \Omega} \cdot n=0, \text { weakly }\right\},
$$

and

$$
\mathbf{J}_{1}=\left\{v \in \mathbf{H}_{0}^{1}, \operatorname{div} v=0\right\} .
$$

We make the following basic assumptions concerning the solution of (1),(2) for our analysis, compare [6],

- condition (A1), concerning the regularity of the domain: The unique solution $u \in \mathbf{J}_{1}$ of the stationary, incompressible Stokes problem with homogeneous boundary data of Dirichlet-type (with the Stokes operator $A \equiv-P_{\mathbf{J}_{0}} \Delta$ ) is already in $\mathbf{J}_{1} \cap \mathbf{H}^{2}$, provided the right-hand side enjoys $f \in \mathbf{L}^{2}$, and satisfies the following stability result,

$$
\|u\|_{2} \leq C\|A u\|
$$


- condition (AD), concerning the regularity of the given data: We suppose the following degrees of regularity for the given data, $\left\{u_{0}, T_{0},\left\{Y_{i}^{0}\right\}_{i=1}^{N}\right\}$

$$
u_{0}, T_{0},\left\{Y_{i}^{0}\right\}_{i=1}^{N} \in \mathbf{H}_{0}^{1} \cap \mathbf{H}^{2} .
$$

The given function $f_{0}$ is supposed to be affine.

- condition (A3), concerning the existence of a strong solution: Given $t_{M+1}>0$, suppose that there exists a strong solution $\left\{u, p, T,\left\{Y_{i}\right\}_{i=1}^{N}\right\} \in L^{\infty}\left(0, t_{M+1} ; \mathcal{X}\right)$, with the space $\mathcal{X}$ defined below.

Note that the existence of a strong solution can only be verified locally in time, with the length of the time interval $\left[0, t_{M+1}\right]$ depending on the parameters of the given problem.

Subsequently, we will make use of the following notation: let $L^{2}(\Omega), H^{r}(\Omega)$, and $H_{0}^{r}(\Omega), r$ an integer, be the standard Lebesgue and Sobolev spaces, see [5] for details. These spaces are endowed with the standard scalar products and their induced norms $\|\cdot\|_{r}$. Further, $H^{-r}(\Omega)$ is the space that is dual to $H^{r}(\Omega) \cap H_{0}^{1}(\Omega)$. $L_{0}^{2}(\Omega)$ is the subspace of $L^{2}(\Omega)$ consisting of functions with vanishing spatial average, which is isomorphic to $L^{2}(\Omega) / \mathrm{R}$. The spaces of vector-valued functions will be indicated with boldface letters, for instance $\mathbf{H}_{0}^{1} \equiv\left(H_{0}^{1}\right)^{d}$, for $d=2,3$.

Due to the evolutionary character of the problem, let $L^{p}\left(0, t_{M+1} ; X\right)$ be the space of functions $\phi=\phi(x, t)$ s.t. holds: the map $t \mapsto\|\phi(t)\|_{X}^{p}, t \in\left[0, t_{M+1}\right]$ is measurable almost everywhere, and $\int_{0}^{t_{M+1}}\|\phi(s)\|_{X}^{p} d s<\infty$, for $1 \leq q<\infty$ and $X$ a Banach space. For the case $p=\infty$, we require the property $\sup _{0 \leq s \leq t_{M+1}}\|\phi(s)\|_{X}<\infty$ to be satisfied. Correspondingly, we define $C\left(0, t_{M+1} ; X\right)$ to be the space of functions $\phi=\phi(x, t)$, s.t. the map $t \mapsto\|\phi(t)\|_{X}$ is continuous, for all $t \in\left[0, t_{M+1}\right]$, and $\max _{0 \leq s \leq t_{M+1}}\|\phi(s)\|_{X}<\infty$. - In the following, we make frequent use of the difference quotient defined by $d_{t} \phi^{m+1}:=\frac{1}{k}\left\{\phi^{m+1}-\phi^{m}\right\}$. Further, we employ the spaces $\ell^{p}\left(0, t_{M+1} ; X\right)$, for $1 \leq p<\infty$, which is the space of functions $\left\{\phi^{m+1}\right\}_{m=0}^{M}$, with bounded norm $\left(k \sum_{m=0}^{M}\left\|\phi^{m+1}\right\|_{X}^{p}\right)^{1 / p}$, for the time-step $k=t_{m+1}-t_{m}$. For the case $p=\infty$, functions $\left\{\phi^{m+1}\right\}_{m=0}^{M}$ need to satisfy $\max _{0 \leq m \leq M}\left\|\phi^{m+1}\right\|_{X}<\infty$. Finally, we employ the notations $\tau(s) \equiv \min \{1, s\}$ and $\tau_{m+1} \equiv \min \left\{1, t_{m+1}\right\}$.

As already mentioned, the analysis of scheme (4) through (7) is split in an investigation of its stability and approximation properties. In order to abbreviate the notational effort, let us introduce the following denomination: Given the quadruple $\left\{a_{i}^{m+1}\right\}_{i=1}^{4} \in \mathcal{X}$ with

$$
\mathcal{X}:=\left(\mathbf{H}_{0}^{1} \cap \mathbf{H}^{2}\right) \times H^{1} / \mathrm{R} \times\left(H_{0}^{1} \cap H^{2}\right) \times \prod_{i=1}^{N}\left(H_{0}^{1} \cap H^{2}\right) .
$$

We then say that

1. the quadruple $\left\{a_{i}^{m+1}\right\}_{i=1}^{4} \in \mathcal{X}$ satisfies Property (P1), provided the following a-priori 
statements are satisfied, for $i \in\{1,3,4\}$,

$$
k \sum_{m=1}^{M}\left\|d_{t} a_{i}^{m+1}\right\|_{1}^{2}+\max _{1 \leq m \leq M}\left\{\left\|d_{t} a_{i}^{m+1}\right\|^{2}+\left\|a_{i}^{m+1}\right\|_{2}^{2}+\left\|\nabla a_{2}^{m+1}\right\|^{2}\right\} \leq C .
$$

2. The quadruple $\left\{a_{i}^{m+1}\right\}_{i=1}^{4} \in \mathcal{X}$ satisfies Property (PD), if the following approximation properties are satisfied:

$$
\begin{aligned}
& \max _{0 \leq m \leq M}\left\{\left\|u\left(t_{m+1}\right)-a_{1}^{m+1}\right\|+\left\|p\left(t_{m+1}\right)-a_{2}^{m+1}\right\|_{-1}+\left\|T\left(t_{m+1}\right)-a_{3}^{m+1}\right\|\right. \\
& \quad+\left\|Y\left(t_{m+1}\right)-a_{4}^{m+1}\right\|+\sqrt{k}\left(\left\|u\left(t_{m+1}\right)-a_{1}^{m+1}\right\|_{1}+\left\|p\left(t_{m+1}\right)-a_{2}^{m+1}\right\|\right. \\
& \left.\left.\quad+\left\|T\left(t_{m+1}\right)-a_{3}^{m+1}\right\|_{1}+\left\|Y\left(t_{m+1}\right)-a_{4}^{m+1}\right\|_{1}\right)\right\} \leq C k,
\end{aligned}
$$

where $Y:=\left\{Y_{i}\right\}_{i=1}^{N}$. We are now in a position to formulate the main result that states optimal convergence behavior of the solution of (4) through (7).

Theorem 2.1 Suppose the basic assumptions (A1), (AD), (A3) to be valid. Then, the solution $\left\{u^{m+1}, p^{m+1}, T^{m+1},\left\{Y_{i}^{m+1}\right\}_{i=1}^{N}\right\} \in \mathcal{X}$ of scheme (4) through (7) satisfies the properties (P1) and (PD), for sufficiently small time-steps $k \leq k_{0}\left(t_{M+1}\right)$.

As we see from property (P2), the pressure approximation quality is worse than corresponding statements for the remaining quantities, measured in the $l^{\infty}\left(0, t_{M+1} ; \mathcal{X}\right)$-norm. This reflects arising boundary layers that are due to the prescription of unphysical, homogeneous pressure data in (8) for the computed pressure iterates. This observation is well-understood in the Navier-Stokes context, see section 4 for further details. Furthermore, this perturbation of the pressure iterates also affects the gradient velocity field, and the question is whether there is also a crucial impact on concentration and temperature iterates from the projection step (5). Surprisingly, it turns out that the answer depends heavily on the dimension $d$ of the problem. In fact, in the case $d=3$ there is a significant impact on the temperature approximation and the mass fractions, caused by the projection step (5).

Corollary 2.1 Suppose the conditions of Theorem 2.1 to be valid. Then, the approximates $\left\{T^{m+1},\left\{Y_{i}^{m+1}\right\}_{i=1}^{N}\right\}$ satisfy the improved estimates

1. in two space dimensions (i.e., $d=2$ ), for all $\gamma>0$, and $\lim _{\gamma \rightarrow 0} C_{\gamma}=\infty$ :

$$
\max _{0 \leq m \leq M} \sqrt{\tau_{m+1}}\left\{\left\|T\left(t_{m+1}\right)-a_{3}^{m+1}\right\|_{1}+\left\|Y\left(t_{m+1}\right)-a_{4}^{m+1}\right\|_{1}\right\} \leq C_{\gamma} k^{1-\gamma},
$$

2. in three space dimensions (i.e., $d=3$ ):

$$
\max _{0 \leq m \leq M} \sqrt{\tau_{m+1}}\left\{\left\|T\left(t_{m+1}\right)-a_{3}^{m+1}\right\|_{1}+\left\|Y\left(t_{m+1}\right)-a_{4}^{m+1}\right\|_{1}\right\} \leq C k^{3 / 4} .
$$


3 Outline of the Proof of Theorem 2.1. In order to understand the ongoing error mechanisms in the numerical model (4) through (7), we will propose several auxiliary problems in the following that will be subject to investigation in the subsequent sections. Each of the presented auxiliary problems is formulated to identify and analyze one of the present error sources inherent to (4) through (7). It is our goal to verify the properties (P1) and (P2) for each single problem.

In the following formulations, we omit to write down the boundary conditions and the initial data if they coincide with those given in Lemma 5.1.

1st auxiliary problem: This problem is proposed to study the impact of implicit timediscretization effects.

For initial data $\left\{u^{0}, T^{0},\left\{Y_{i}^{0}\right\}_{i=1}^{N}\right\}$ given in (1), determine $\left\{u_{a}^{m+1}, p_{a}^{m+1}, T_{a}^{m+1},\left\{Y_{a, i}^{m+1}\right\}_{i=1}^{N}\right\} \in$ $\mathcal{X}$ as the solution of

$$
\begin{aligned}
& d_{t} u_{a}^{m+1}-\Delta u_{a}^{m+1}+\left(u_{a}^{m+1} \cdot \nabla\right) u_{a}^{m+1}+\nabla p_{a}^{m+1}=f_{0}\left(T_{a}^{m+1}\right), \\
& \operatorname{div} u_{a}^{m+1}=0 \\
& d_{t} T_{a}^{m+1}-\Delta T_{a}^{m+1}+\left(u_{a}^{m+1} \cdot \nabla\right) T_{a}^{m+1}=-\sum_{i=1}^{N} h_{i} W_{i}\left(\left\{Y_{a, i}^{m+1}\right\}_{i=1}^{N}, T_{a}^{m+1}\right) . \\
& d_{t} Y_{a, i}^{m+1}-\Delta Y_{a, i}^{m+1}+\left(u_{a}^{m+1} \cdot \nabla\right) Y_{a, i}^{m+1}=W_{i}\left(\left\{Y_{a, i}^{m+1}\right\}_{i=1}^{N}, T_{a}^{m+1}\right) .
\end{aligned}
$$

The result of the investigation is given in Lemma 6.1 .

2nd auxiliary problem: In this auxiliary problem, we analyze the explicit coupling of temperature phenomena and the momentum equation.

For initial data $\left\{u^{0}, T^{0},\left\{Y_{i}^{0}\right\}_{i=1}^{N}\right\}$ given in (1), determine $\left\{u_{b}^{m+1}, p_{b}^{m+1}, T_{b}^{m+1},\left\{Y_{b, i}^{m+1}\right\}\right\}_{i=1}^{N} \in$ $\mathcal{X}$ being the solution of

$$
\begin{aligned}
& d_{t} u_{b}^{m+1}-\Delta u_{b}^{m+1}+\left(u_{b}^{m+1} \cdot \nabla\right) u_{b}^{m+1}+\nabla p_{b}^{m+1}=f_{0}\left(T_{b}^{m}\right), \\
& \operatorname{div} u_{b}^{m+1}=0 \\
& d_{t} T_{b}^{m+1}-\Delta T_{b}^{m+1}+\left(u_{b}^{m+1} \cdot \nabla\right) T_{b}^{m+1}=-\sum_{i=1}^{N} h_{i} W_{i}\left(\left\{Y_{b, i}^{m+1}\right\}_{i=1}^{N}, T_{b}^{m+1}\right) . \\
& d_{t} Y_{b, i}^{m+1}-\Delta Y_{b, i}^{m+1}+\left(u_{b}^{m+1} \cdot \nabla\right) Y_{b, i}^{m+1}=W_{i}\left(\left\{Y_{b, i}^{m+1}\right\}_{i=1}^{N}, T_{b}^{m+1}\right) .
\end{aligned}
$$

We refer to Lemma 6.2 for corresponding statements of convergence and stability.

3rd auxiliary problem: The investigation of the influence of an "explicit treatment" of the convective part is the reason for the following auxiliary problem:

For initial data $\left\{u^{0}, T^{0},\left\{Y_{i}^{0}\right\}_{i=1}^{N}\right\}$ given in (1), determine $\left\{u_{c}^{m+1}, p_{c}^{m+1}, T_{c}^{m+1},\left\{Y_{c, i}^{m+1}\right\}_{i=1}^{N}\right\} \in$ 
$\mathcal{X}$ being the solution of

$$
\begin{aligned}
& d_{t} u_{c}^{m+1}-\Delta u_{c}^{m+1}+\left(u_{c}^{m} \cdot \nabla\right) u_{c}^{m+1}+\nabla p_{c}^{m+1}=f_{0}\left(T_{c}^{m}\right), \\
& \operatorname{div} u_{d}^{m+1}=0, \\
& d_{t} T_{c}^{m+1}-\Delta T_{c}^{m+1}+\left(u_{c}^{m} \cdot \nabla\right) T_{c}^{m+1}=-\sum_{i=1}^{N} h_{i} W_{i}\left(\left\{Y_{c, i}^{m+1}\right\}_{i=1}^{N}, T_{c}^{m+1}\right) . \\
& d_{t} Y_{c, i}^{m+1}-\Delta Y_{c, i}^{m+1}+\left(u_{c}^{m} \cdot \nabla\right) Y_{c, i}^{m+1}=W_{i}\left(\left\{Y_{c, i}^{m+1}\right\}_{i=1}^{N}, T_{c}^{m+1}\right) .
\end{aligned}
$$

The results of this analysis are summarized in Lemma 6.3 .

4th auxiliary problem: This auxiliary problem deals with the decoupling of the chemical part, i.e., temperature and concentrations.

For initial data $\left\{u^{0}, T^{0},\left\{Y_{i}^{0}\right\}_{i=1}^{N}\right\}$ given in (1), determine $\left\{u_{d}^{m+1}, p_{d}^{m+1}, T_{d}^{m+1},\left\{Y_{d, i}^{m+1}\right\}_{i=1}^{N}\right\} \in$ $\mathcal{X}$ being the solution of

$$
\begin{aligned}
& d_{t} u_{d}^{m+1}-\Delta u_{d}^{m+1}+\left(u_{d}^{m} \cdot \nabla\right) u_{d}^{m+1}+\nabla p_{d}^{m+1}=f_{0}\left(T_{d}^{m}\right), \\
& \operatorname{div} u_{d}^{m+1}=0, \\
& d_{t} T_{d}^{m+1}-\Delta T_{d}^{m+1}+\left(u_{d}^{m} \cdot \nabla\right) T_{d}^{m+1}=-\sum_{i=1}^{N} h_{i} W_{i}\left(\left\{Y_{d, i}^{m}\right\}_{i=1}^{N}, T_{d}^{m+1}\right) . \\
& d_{t} Y_{d, i}^{m+1}-\Delta Y_{d, i}^{m+1}+\left(u_{d}^{m} \cdot \nabla\right) Y_{d, i}^{m+1}=W_{i}\left(\left\{Y_{d, i}^{m+1}\right\}_{i=1}^{N}, T_{d}^{m}\right) .
\end{aligned}
$$

We refer to Lemma 6.4 for a summary of the related analysis.

5th auxiliary problem: In order to study the impact of the projection scheme of Chorin, we will be dealing with the following problem:

For initial data $\left\{u^{0}, T^{0},\left\{Y_{i}^{0}\right\}_{i=1}^{N}\right\}$ given in (1), and $\left\{T_{d}^{m},\left\{Y_{d, i}^{m}\right\}_{i=1}^{N}\right\}_{m=0}^{M}$ determined by (12), compute iterates $\left\{u_{e}^{m+1}, p_{e}^{m+1}\right\} \in \mathbf{H}_{0}^{1} \times H^{1} / \mathrm{R}$ being the solution of

$$
\begin{aligned}
& d_{t} u_{e}^{m+1}-\Delta u_{e}^{m+1}+\left(P_{\mathbf{J}_{0}} u_{e}^{m} \cdot \nabla\right) u_{e}^{m+1}+\nabla p_{e}^{m}=f_{0}\left(T_{d}^{m}\right), \\
& \operatorname{div} u_{e}^{m+1}-k \Delta p_{e}^{m+1}=0,\left.\quad \partial_{n} p_{e}^{m+1}\right|_{\partial \Omega}=0 .
\end{aligned}
$$

Corresponding statements on the solution behavior will be given in Lemma 6.5.

6th auxiliary problem: We will finally study the recoupling of the "flow part" with the "chemical part".

For initial data $\left\{u^{0}, T^{0},\left\{Y_{i}^{0}\right\}_{i=1}^{N}\right\}$ given in (1), determine $\left\{u_{f}^{m+1}, p_{f}^{m+1}, T_{f}^{m+1},\left\{Y_{f, i}^{m+1}\right\}\right\}_{i=1}^{N} \in$ $\mathcal{X}$ being the solution of

$$
\begin{aligned}
& d_{t} u_{f}^{m+1}-\Delta u_{f}^{m+1}+\left(P_{\mathbf{J}_{0}} u_{f}^{m} \cdot \nabla\right) u_{f}^{m+1}+\nabla p_{f}^{m}=f_{0}\left(T_{f}^{m}\right), \\
& \operatorname{div} u_{f}^{m+1}-k \Delta p_{f}^{m+1}=0,\left.\quad \partial_{n} p_{f}^{m+1}\right|_{\partial \Omega}=0, \\
& d_{t} T_{f}^{m+1}-\Delta T_{f}^{m+1}+\left(u_{f}^{m} \cdot \nabla\right) T_{f}^{m+1}=-\sum_{i=1}^{N} h_{i} W_{i}\left(\left\{Y_{f, i}^{m}\right\}_{i=1}^{N}, T_{f}^{m+1}\right) . \\
& d_{t} Y_{f, i}^{m+1}-\Delta Y_{f, i}^{m+1}+\left(u_{f}^{m} \cdot \nabla\right) Y_{f, i}^{m+1}=W_{i}\left(\left\{Y_{f, i}^{m+1}\right\}_{i=1}^{N}, T_{f}^{m}\right) .
\end{aligned}
$$


Lemma 6.6 provides the results of the corresponding analysis.

The combination of the Lemmata 6.1 through 6.6 provides the proof of Theorem 2.1.

4 Chorin's projection method. In 1968, Chorin has proposed a splitting algorithm to reduce the computational effort in CFD simulations, see [3, 4]. The idea is to decouple the computation in each iteration step by first calculating a guess $\tilde{u}^{m+1} \in \mathbf{H}_{0}^{1}$ for the new velocity and then projecting it in the space of divergence free functions by means of calculating a pressure approximate as a solution of a Laplace-Neumann problem.

Let us fix notation and the problem setting where this projection method was originally applied to. Assume $\Omega \subset \mathrm{R}^{3}$ to be a bounded domain where an incompressible fluid flow with constant viscosity $\nu>0$ is in, driven by a given external force $f=f(x, t)$. The equations governing the flow are the following ones,

$$
\begin{aligned}
& u_{t}-\nu \Delta u+(u \cdot \nabla) u+\nabla p=f, \\
& \operatorname{div} u=0,
\end{aligned}
$$

together with initial and boundary value data,

$$
u(0)=u_{0} \in \mathbf{J}_{1} \cap \mathbf{H}^{2},\left.\quad u\right|_{\partial \Omega}=0 .
$$

Now, Chorin's method reads as follows:

1. Start with an approximate initial guess $u^{0} \approx u_{0}$.

2. For $m \geq 0$, find $\tilde{u}^{m+1}$ as the solution of

$$
\begin{aligned}
& \frac{1}{k}\left\{\tilde{u}^{m+1}-u^{m}\right\}-\nu \Delta \tilde{u}^{m+1}+\left(u^{m} \cdot \nabla\right) \tilde{u}^{m+1}=f^{m+1}, \\
& \left.\tilde{u}^{m+1}\right|_{\partial \Omega}=0 .
\end{aligned}
$$

3. Provided with $\tilde{u}^{m+1}$, determine the tuple $\left\{u^{m+1}, p^{m+1}\right\}$ as the solution of

$$
\begin{aligned}
& \frac{1}{k}\left\{u^{m+1}-\tilde{u}^{m+1}\right\}+\nabla p^{m+1}=0, \\
& \operatorname{div} u^{m+1}=0,\left.\quad u^{m+1}\right|_{\partial \Omega} \cdot n=0 .
\end{aligned}
$$

The projection step (18) can be reformulated through application of the div operator, leading to a Laplace-Neumann problem for the pressure,

$$
-\Delta p^{m+1}=-\frac{1}{k} \operatorname{div} \tilde{u}^{m+1},\left.\quad \partial_{n} p^{m+1}\right|_{\partial \Omega}=0 .
$$

Now, the projection method of Chorin is the following: In each iteration step, calculate a guess $\tilde{u}^{m+1}$ from (17) and then determine $p^{m+1}$ from (19). Finally, calculate $u^{m+1}$ from (18) through a simple algebraic update. 
The key observation to start an error analysis for this method is its reinterpretation as a semi-explicit pressure stabilization method. For the purpose of this work, we recall the main statements of stability of the solution and its approximation features with respect to the solution of (15) in the subsequent lemma, see [13].

Lemma 4.1 Suppose $\left\{\tilde{u}^{m+1}, p^{m+1}\right\}$ to be the (semi-)discrete solution of Chorin's method (17), (18), whereas $\left\{u\left(t_{m+1}\right), p\left(t_{m+1}\right)\right\}$ is the solution of the Navier-Stokes equations (15) at time $0<t_{m+1} \leq t_{M+1}$. Assume the basic assumptions (A1), (AD) and (A3) be valid, for a given function $f=f_{0}$. Then, for sufficient small time-steps $k \leq k_{0}\left(t_{M+1}\right)$, there exists a constant $C$ which only depends on the given data of the problem, such that the following

1. convergence estimates hold true:

$$
\begin{aligned}
& \max _{0 \leq m \leq M}\left\{\left\|u\left(t_{m+1}\right)-\tilde{u}^{m+1}\right\|+\tau_{m+1}\left\|p\left(t_{m+1}\right)-p^{m+1}\right\|_{-1}\right\} \leq C k, \\
& \max _{0 \leq m \leq M}\left\{\left\|u\left(t_{m+1}\right)-\tilde{u}^{m+1}\right\|_{1}+\sqrt{\tau_{m+1}}\left\|p\left(t_{m+1}\right)-p^{m+1}\right\|\right\} \leq C \sqrt{k} .
\end{aligned}
$$

2. stability results hold true:

$$
\max _{0 \leq m \leq M}\left\{\left\|d_{t} \tilde{u}^{m+1}\right\|+\left\|\tilde{u}^{m+1}\right\|_{2}+\left\|\nabla p^{m+1}\right\|\right\}+k \sum_{m=0}^{M}\left\|\nabla d_{t} \tilde{u}^{m+1}\right\|^{2} \leq C .
$$

5 A priori Analysis of the Chemically Flow Problem. This section is devoted to the presentation of striking a priori bounds for the solution of system (1),(2), as well as to the presentation of energy arguments that will continuously be employed in the following sections. Let us recall that the well-posedness of the problem has been verified in [9] and [16]. Note that standard regularity results apply, furnishing $L^{\infty}\left(0, t_{M+1}, H_{0}^{1} \cap H^{2}\right)$-regularity of velocity field $u$, temperature $T$ and mass fractions $\left\{Y_{i}\right\}_{i=1}^{N}$, thanks to the parabolic character of the equations and the regularity of the given data. We omit the elaboration of these (sketchy) standard arguments, since they can be given analogously to those that guarantee the existence, uniqueness and regularity of (weak and strong) solutions for the incompressible Navier-Stokes equations, see $[17,16]$.

Lemma 5.1 Assume $\left\{u, p, T,\left\{Y_{i}\right\}_{i=1}^{N}\right\} \in L^{\infty}\left(0, t_{M+1} ; \mathcal{X}\right)$ to be the solution of (1), (2), and the given data to satisfy the assumptions (A1), (AD), (A3). Then, the following a priori statements are satisfied,

$$
\begin{aligned}
& \sup _{\left[0, t_{M+1}\right]}\left\{\|u\|_{2}+\|p\|_{1}+\|T\|_{2}+\sum_{i=1}^{N}\left\|Y_{i}\right\|_{2}\right\} \leq C, \\
& \sup _{\left(0, t_{M+1}\right]}\left\{\left\|u_{t}\right\|+\left\|T_{t}\right\|+\sum_{i=1}^{N}\left\|Y_{i, t}\right\|\right\}^{2}+\int_{0}^{t_{M+1}}\left\{\left\|u_{t}(s)\right\|_{1}^{2}+\left\|T_{t}(s)\right\|_{1}^{2}+\sum_{i=1}^{N}\left\|Y_{i, t}(s)\right\|_{1}^{2}\right\} d s \\
& \quad+\int_{0}^{t_{M+1}}\left\{\left\|u_{t t}(s)\right\|_{-1}^{2}+\left\|T_{t t}(s)\right\|_{-1}^{2}+\sum_{i=1}^{N}\left\|Y_{i, t t}(s)\right\|_{-1}^{2}\right\} d s \leq C,
\end{aligned}
$$

where $C$ is a constant that depends on the given data of the problem. 
Proof. The verification can be accomplished by means of simple energy arguments: we observe that the construction of each equation is of diffusion-convection-reaction type. The transfer of information is solely accomplished through the leading term in the convection part and the reaction terms, in particular with "lower order" operators. Therefore, testing the equations in (1) successively with $\left\{-\Delta u,-\Delta T,\left\{-\Delta Y_{i}\right\}_{i=1}^{N}\right\}$ and summing give

$$
\begin{aligned}
& \frac{1}{2} d_{t}\left\{\|u\|_{1}^{2}+\|T\|_{1}^{2}+\sum_{i=1}^{N}\left\|Y_{i}\right\|_{1}^{2}\right\}+\|u\|_{2}^{2}+\|T\|_{2}^{2}+\sum_{i=1}^{N}\left\|Y_{i}\right\|_{2}^{2} \\
& \leq C\left\{\|\nabla u\|^{6}+\|\nabla u\|^{4}\|\nabla T\|^{2}+\|\nabla u\|^{4} \sum_{i=1}^{N}\left\|\nabla Y_{i}\right\|^{2}+\|T\|^{2}\right\}
\end{aligned}
$$

If we apply Gronwall's inequality, we are lead to the first result

$$
\begin{aligned}
& \sup _{\left[0, t_{M+1}\right]}\left\{\|u\|_{1}^{2}+\|T\|_{1}^{2}+\sum_{i=1}^{N}\left\|Y_{i}\right\|_{1}^{2}\right\} \\
& \quad+\int_{0}^{t_{M+1}}\left\{\|u(s)\|_{2}^{2}+\|T(s)\|_{2}^{2}+\sum_{i=1}^{N}\left\|Y_{i}(s)\right\|_{2}^{2}\right\} d s \leq C .
\end{aligned}
$$

We now differentiate system (1) in time (in a weak sense) and finally test it with $\left\{u_{t}, T_{t},\left\{Y_{i, t}\right\}_{i=1}^{N}\right\}$. We can make use of the skew-symmetricity rule that holds for the trilinear form

$$
b\left(\phi ; \psi_{1}, \psi_{2}\right):=\int_{\Omega}(\phi \cdot \nabla) \psi_{1} \psi_{2} d \Omega
$$

which implies

$$
b\left(\phi ; \psi_{1}, \psi_{2}\right)=-b\left(\phi ; \psi_{2}, \psi_{1}\right), \quad \forall\left\{\phi, \psi_{1}, \psi_{2}\right\} \in \mathbf{J}_{0} \times\left(\mathbf{H}_{0}^{1}\right)^{2} .
$$

From this and the Lipschitz continuity of $\left\{W_{i}\right\}_{i=1}^{N}$, we end up with

$$
\begin{gathered}
\frac{1}{2} d_{t}\left\{\left\|u_{t}\right\|^{2}+\left\|T_{t}\right\|^{2}+\sum_{i=1}^{N}\left\|Y_{i, t}\right\|^{2}\right\}+\left\|u_{t}\right\|_{1}^{2}+\left\|T_{t}\right\|_{1}^{2}+\sum_{i=1}^{N}\left\|Y_{i, t}\right\|_{1}^{2} \\
\leq C\left\{\left\|u_{t}\right\|^{2}+\left\|T_{t}\right\|^{2}+\sum_{i=1}^{N}\left\|Y_{i, t}\right\|^{2}+\left\|u_{t}\right\|^{2}\|u\|_{1}\|u\|_{2}\right. \\
\left.+\left\|u_{t}\right\|^{2}\|T\|_{1}\|T\|_{2}+\left\|u_{t}\right\|^{2} \sum_{i=1}^{N}\left\|Y_{i}\right\|_{1}\left\|Y_{i}\right\|_{2}\right\} .
\end{gathered}
$$

From this inequality, we shall integrate over the time interval $\left[0, t_{M+1}\right]$. Owing to (20), we obtain pointwise in time a priori statements for the quantities $\left\{u_{t}, T_{t},\left\{Y_{i, t}\right\}_{i=1}^{N}\right\}$ in the $L^{2}$ product norm. - Now, in order to verify the remaining statements that are formulated in 
the lemma, we turn back to the equations (1): The terms $\left\{\|\Delta u\|,\|\Delta T\|, \sum_{i=1}^{N}\left\|\Delta Y_{i}\right\|\right\}$ can now be easily bounded by quantities that we have already under control. - The validity of the boundedness of the remaining quantities is now an immediate consequence of the previous results.

Remark 5.1 For subsequent studies, we also need a-priori statements for higher timederivatives of velocity field, temperature function, and mass fraction functions. As it is known from previous works, see $[6,8]$, this involves time-weights to control initial rough perturbances. We skip the verification of the following a-priori results, refering to the cited literature for the needed standard energy arguments,

$$
\begin{aligned}
& \int_{0}^{t_{M+1}} \tau(s)\left\{\left\|u_{t}(s)\right\|_{2}^{2}+\left\|T_{t}(s)\right\|_{2}^{2}+\sum_{i=1}^{N}\left\|Y_{i, t}(s)\right\|_{2}^{2}\right\} d s \\
& \quad+\int_{0}^{t_{M+1}} \tau(s)\left\{\left\|u_{t t}(s)\right\|^{2}+\left\|T_{t t}(s)\right\|^{2}+\sum_{i=1}^{N}\left\|Y_{i, t t}(s)\right\|^{2}\right\} d s \leq C .
\end{aligned}
$$

In subsequent considerations, we shall make frequent use of energy type arguments without presenting them in detailed way. - The following sections are now devoted to analyze the auxiliary problems proposed in section 3 .

\section{Analysis of the Auxiliary Problems.}

6.1 The auxiliary problem (9). The presentation of a consistence analysis is the goal of this section. We will study the approximation properties of the fully implicit timediscretization scheme (9). The main result presented in this section is summarized in the following lemma.

Lemma 6.1 Assume the assumptions (A1), (A2) and (A3) to be valid for the solution of (1), (2). Then, the solution $\left\{u_{a}^{m+1}, p_{a}^{m+1}, T_{a}^{m+1},\left\{Y_{i, a}^{m+1}\right\}_{i=1}^{N}\right\} \in \mathcal{X}$ of scheme (9) satisfies the properties (P1) and (PD), for sufficiently small time-steps $k \leq k_{0}\left(t_{M+1}\right)$.

Proof. We omit the verification of the statements given in $(\mathrm{P} 1)$ for the problem, since they can be immediately verified by means of arguments that are analogous to those presented in the proof of Lemma 5.1, causing no further difficulties. - In order to verify the property (P2), we subtract the equations (1) and (9). In the following, we will make use of the following abbreviative notation,

$$
\begin{array}{ll}
e^{m+1}:=u\left(t_{m+1}\right)-u_{a}^{m+1}, & \eta^{m+1}:=p\left(t_{m+1}\right)-p_{a}^{m+1} \\
\mathcal{T}^{m+1}:=T\left(t_{m+1}\right)-T_{a}^{m+1}, & \mathcal{Y}_{i}^{m+1}:=Y\left(t_{m+1}\right)-Y_{a, i}^{m+1} .
\end{array}
$$


Then, the resulting error equations are as follows,

$$
\begin{aligned}
& d_{t} e^{m+1}-\Delta e^{m+1}+\left(u\left(t_{m+1}\right) \cdot \nabla\right) e^{m+1}+\left(e^{m+1} \cdot \nabla\right) u_{a}^{m+1} \\
& \quad+\nabla \eta^{m+1}=r^{m+1}(u)+\left[f_{0}\left(T\left(t_{m+1}\right)\right)-f_{0}\left(T_{a}^{m+1}\right)\right], \\
& \operatorname{div} e^{m+1}=0, \\
& d_{t} \mathcal{T}^{m+1}-\Delta \mathcal{T}^{m+1}+\left(u\left(t_{m+1}\right) \cdot \nabla\right) \mathcal{T}^{m+1}+\left(e^{m+1} \cdot \nabla\right) T_{a}^{m+1} \\
& \quad=r^{m+1}(T)-\sum_{i=1}^{N} h_{i}\left[W_{i}\left(Y_{i}\left(t_{m+1}\right), T\left(t_{m+1}\right)\right)-W_{i}\left(Y_{a, i}^{m+1}, T_{a}^{m+1}\right)\right], \\
& d_{t} \mathcal{Y}_{i}^{m+1}-\Delta \mathcal{Y}_{i}^{m+1}+\left(u\left(t_{m+1}\right) \cdot \nabla\right) \mathcal{Y}_{i}^{m+1}+\left(e^{m+1} \cdot \nabla\right) Y_{a, i}^{m+1} \\
& \quad=r^{m+1}\left(Y_{i}\right)+\left[W_{i}\left(Y_{i}\left(t_{m+1}, T\left(t_{m+1}\right)\right)-W_{i}\left(Y_{a, i}^{m+1}, T_{a}^{m+1}\right)\right],\right.
\end{aligned}
$$

where

$$
r^{m+1}(\phi):=-\frac{1}{k} \int_{t_{m}}^{t_{m+1}}\left(s-t_{m}\right) \phi_{t t}(s) d s .
$$

We start the analysis of (22), testing the first equation in (22) with $e^{m+1}$ and the subsequent ones with $\mathcal{T}^{m+1}$ and $\mathcal{Y}_{i}^{m+1}$. We finally obtain

$$
\begin{aligned}
& \frac{1}{2} d_{t}\left\{\left\|e^{m+1}\right\|^{2}+\left\|\mathcal{T}^{m+1}\right\|^{2}+\sum_{i=1}^{N}\left\|\mathcal{Y}_{i}^{m+1}\right\|^{2}\right\} \\
& +\left\|\nabla e^{m+1}\right\|^{2}+\left\|\nabla \mathcal{T}^{m+1}\right\|^{2}+\sum_{i=1}^{N}\left\|\nabla \mathcal{Y}_{i}^{m+1}\right\|^{2} \\
& \leq C\left\{\left\|r^{m+1}(u)\right\|_{-1}^{2}+\left\|r^{m+1}(T)\right\|_{-1}^{2}+\sum_{i=1}^{N}\left\|r^{m+1}\left(Y_{i}\right)\right\|_{-1}^{2}\right. \\
& \left.\quad+\left\|e^{m+1}\right\|^{2}+\left\|\mathcal{T}^{m+1}\right\|^{2}+\sum_{i=1}^{N}\left\|\mathcal{Y}_{i}^{m+1}\right\|^{2}\right\} .
\end{aligned}
$$

Thanks to Lemma 5.1, we have

$$
k \sum_{m=0}^{M}\left\|r^{m+1}(\phi)\right\|_{-1}^{2} \leq C k^{-1} \sum_{m=0}^{M} \int_{t_{m}}^{t_{m+1}}\left(s-t_{m}\right)^{2} d s \int_{t_{m}}^{t_{m+1}}\left\|\phi_{t t}(s)\right\|_{-1}^{2} d s \leq C k^{2} .
$$

Thus, using the discrete Gronwall Lemma in (24), we have succeeded in proving error statement (P2). 
6.2 The auxiliary problem (10). We will now focus on the perturbation effect that an explicit treatment of the temperature function on the right hand side of the first equation in (10) has on the approximation of the solution $\left\{u_{a}^{m+1}, p_{a}^{m+1}, T_{a}^{m+1},\left\{Y_{a, i}^{m+1}\right\}_{i=1}^{N}\right\}$. The proof of the following Lemma is presented in the remainder of this section.

Lemma 6.2 Suppose the basic assumptions (A1), (AD), (A3) to be valid. Then, the solution $\left\{u_{b}^{m+1}, p_{b}^{m+1}, T_{b}^{m+1},\left\{Y_{b, i}^{m+1}\right\}_{i=1}^{N}\right\} \in \mathcal{X}$ of scheme (10) satisfies the properties (P1) and (PQ), provided time-steps $k \leq k_{0}\left(t_{M+1}\right)$ are chosen that are sufficiently small.

Proof. It is sufficient to compare the difference in the solutions of (9) and (10), since (9) inherits the properties under consideration, see Lemma 6.1. - Using the error notation,

$$
\begin{aligned}
& e^{m+1}:=u_{a}^{m+1}-u_{b}^{m+1}, \quad \eta^{m+1}:=p_{a}^{m+1}-p_{b}^{m+1}, \\
& \mathcal{T}^{m+1}:=T_{a}^{m+1}-T_{b}^{m+1}, \quad \mathcal{Y}_{i}^{m+1}:=Y_{a, i}^{m+1}-Y_{b, i}^{m+1},
\end{aligned}
$$

the error equations are then the following ones,

$$
\begin{aligned}
& \left.d_{t} e^{m+1}-\Delta e^{m+1}+\left(u_{a}^{m+1}\right) \cdot \nabla\right) e^{m+1}+\left(e^{m+1} \cdot \nabla\right) u_{b}^{m+1}+\nabla \eta^{m+1} \\
& =\left[f_{0}\left(T_{a}^{m+1}\right)-f_{0}\left(T_{a}^{m}\right)+f_{0}\left(T_{a}^{m}\right)-f_{0}\left(T_{b}^{m}\right)\right], \\
& \operatorname{div} e^{m+1}=0 \text {, } \\
& d_{t} \mathcal{T}^{m+1}-\Delta \mathcal{T}^{m+1}+\left(u_{a}^{m+1} \cdot \nabla\right) \mathcal{T}^{m+1}+\left(e^{m+1} \cdot \nabla\right) T_{b}^{m+1} \\
& =-\sum_{i=1}^{N} h_{i}\left[W_{i}\left(\left\{Y_{a, i}^{m+1}\right\}_{i=1}^{N}, T_{a}^{m+1}\right)-W_{i}\left(\left\{Y_{b, i}^{m+1}\right\}_{i=1}^{N}, T_{b}^{m+1}\right)\right], \\
& d_{t} \mathcal{Y}_{i}^{m+1}-\Delta \mathcal{Y}_{i}^{m+1}+\left(u_{a}^{m+1} \cdot \nabla\right) \mathcal{Y}_{i}^{m+1}+\left(e^{m+1} \cdot \nabla\right) Y_{b, i}^{m+1} \\
& =\left[W_{i}\left(\left\{Y_{a, i}^{m+1}\right\}_{i=1}^{N}, T_{a}^{m+1}\right)-W_{i}\left(\left\{Y_{b, i}^{m+1}\right\}_{i=1}^{N}, T_{b}^{m+1}\right)\right] .
\end{aligned}
$$

For the corresponding error analysis, we can make use of the fact that the $\left\{W_{i}\right\}_{i=1}^{N}$ are all Lipschitz functions and, secondly, the fact that $f_{0}$ is an affine functions. Then, an immediate analysis establishes the properties (P2). - The verification of (P1) can again be done analogously to the proof of Lemma 5.1 and will be omitted therefore.

6.3 The auxiliary problem (11). In here, we investigate the influence of an "explicit treatment" of the flow information in the chemical part. The results of the corresponding error analysis are formulated in the following lemma.

Lemma 6.3 Suppose the basic assumptions (A1),(AD),(A3) to be valid. Then, the solution $\left\{u_{c}^{m+1}, p_{c}^{m+1}, T_{c}^{m+1},\left\{Y_{c, i}^{m+1}\right\}_{i=1}^{N}\right\} \in \mathcal{X}$ of scheme (11) satisfies the properties (P1) and (PQ), for sufficiently small time-steps $k \leq k_{0}\left(t_{M+1}\right)$. 
Proof. Again, we omit the straightforward verification of property (P1) and discuss the validity of property (P2), focusing on the difference in the solution of the systems (10) and (11). By using the error functions

$$
\begin{aligned}
& e^{m+1}:=u_{b}^{m+1}-u_{c}^{m+1}, \quad \eta^{m+1}:=p_{b}^{m+1}-p_{c}^{m+1}, \\
& \mathcal{T}^{m+1}:=T_{b}^{m+1}-T_{c}^{m+1}, \quad \mathcal{Y}_{i}^{m+1}:=Y_{b, i}^{m+1}-Y_{c, i}^{m+1},
\end{aligned}
$$

the corresponding error identities are as follows,

$$
\begin{aligned}
& d_{t} e^{m+1}-\Delta e^{m+1}+\left(u_{b}^{m} \cdot \nabla\right) e^{m+1}+\left(e^{m} \cdot \nabla\right) u_{c}^{m+1}+k\left(d_{t} u_{b}^{m+1} \cdot \nabla\right) u_{b}^{m+1}+\nabla \eta^{m+1} \\
& \quad=\left[f_{0}\left(T_{b}^{m}\right)-f_{0}\left(T_{c}^{m}\right)\right], \\
& \begin{aligned}
& \operatorname{div} e^{m+1}=0 \\
& d_{t} \mathcal{T}^{m+1}-\Delta \mathcal{T}^{m+1}+\left(u_{b}^{m} \cdot \nabla\right) \mathcal{T}^{m+1}+\left(e^{m} \cdot \nabla\right) u_{c}^{m+1}+k\left(d_{t} u_{b}^{m+1} \cdot \nabla\right) u_{b}^{m+1} \\
& \quad=-\sum_{i=1}^{N} h_{i}\left[W_{i}\left(\left\{Y_{b, i}^{m+1}\right\}_{i=1}^{N}, T_{b}^{m+1}\right)-W_{i}\left(\left\{Y_{c, i}^{m+1}\right\}_{i=1}^{N}, T_{c}^{m+1}\right)\right], \\
& d_{t} \mathcal{Y}_{i}^{m+1}-\Delta \mathcal{Y}_{i}^{m+1}+\left(u_{b}^{m} \cdot \nabla\right) \mathcal{Y}_{i}^{m+1}+\left(e^{m} \cdot \nabla\right) Y_{c, i}^{m+1}+k\left(d_{t} u_{b}^{m+1} \cdot \nabla\right) Y_{b, i}^{m+1} \\
& \quad=\left[W_{i}\left(\left\{Y_{b, i}^{m+1}\right\}_{i=1}^{N}, T_{b}^{m+1}\right)-W_{i}\left(\left\{Y_{c, i}^{m+1}\right\}_{i=1}^{N}, T_{c}^{m+1}\right)\right] .
\end{aligned}
\end{aligned}
$$

The energy arguments can again be carried out in a straightforward manner, owing to the validity of Lemma 6.2 , the properties of the functions $f_{0}$ and $\left\{W_{i}\right\}_{i=1}^{N}$, by applying the discrete Gronwall Lemma. This furnishes property (P2).

6.4 The auxiliary problem (12). This step is devoted to analyze the error effect coming from the semi-explicit treatment of the reaction part in the temperature and concentration equations. The preservation of the properties (P1) and (P2) is assured through the next lemma.

Lemma 6.4 Suppose the assumptions (A1),(AD),(A3) to be valid for the solution of (1), (D). Then, the solution $\left\{u_{d}^{m+1}, p_{d}^{m+1}, T_{d}^{m+1},\left\{Y_{d, i}^{m+1}\right\}\right\} \in \mathcal{X}$ of scheme (1D) satisfies the properties (P1) and (PD), for sufficiently small time-steps $k \leq k_{0}\left(t_{M+1}\right)$.

Proof. Again, the verification of property (P1) is straightforward and we move forward to writing down the equations that determine the difference in the solutions of the systems (11) and (12). Setting

$$
\begin{aligned}
& e^{m+1}:=u_{c}^{m+1}-u_{d}^{m+1}, \quad \eta^{m+1}:=p_{c}^{m+1}-p_{d}^{m+1}, \\
& \mathcal{T}^{m+1}:=T_{c}^{m+1}-T_{d}^{m+1}, \quad \mathcal{Y}_{i}^{m+1}:=Y_{c, i}^{m+1}-Y_{d, i}^{m+1},
\end{aligned}
$$


we have the following system of equations,

$$
\begin{aligned}
& d_{t} e^{m+1}-\Delta e^{m+1}+\left(u_{c}^{m} \cdot \nabla\right) e^{m+1}+\left(e^{m} \cdot \nabla\right) u_{d}^{m+1}+\nabla \eta^{m+1}=\left[f_{0}\left(T_{c}^{m}\right)-f_{0}\left(T_{d}^{m}\right)\right] \\
& \operatorname{div} e^{m+1}=0 \\
& d_{t} \mathcal{T}^{m+1}-\Delta \mathcal{T}^{m+1}+\left(u_{c}^{m} \cdot \nabla\right) \mathcal{T}^{m+1}+\left(e^{m} \cdot \nabla\right) T_{d}^{m+1} \\
& \quad-\sum_{i=1}^{N} h_{i}\left[W_{i}\left(\left\{Y_{c, i}^{m+1}\right\}_{i=1}^{N}, T_{c}^{m+1}\right)-W_{i}\left(\left\{Y_{c, i}^{m}\right\}_{i=1}^{N}, T_{c}^{m+1}\right)\right. \\
& \left.\quad+W_{i}\left(\left\{Y_{c, i}^{m}\right\}_{i=1}^{N}, T_{c}^{m+1}\right)-W_{i}\left(\left\{Y_{d, i}^{m}\right\}_{i=1}^{N}, T_{d}^{m+1}\right)\right] \\
& d_{t} \mathcal{Y}_{i}^{m+1}-\Delta \mathcal{Y}_{i}^{m+1}+\left(u_{c}^{m} \cdot \nabla\right) \mathcal{Y}_{i}^{m+1}+\left(e^{m} \cdot \nabla\right) Y_{d, i}^{m+1} \\
& \quad=\left[W_{i}\left(\left\{Y_{c, i}^{m}\right\}_{i=1}^{N}, T_{c}^{m+1}\right)-W_{i}\left(\left\{Y_{c, i}^{m}\right\}_{i=1}^{N}, T_{c}^{m}\right)\right. \\
& \left.\quad+W_{i}\left(\left\{Y_{c, i}^{m}\right\}_{i=1}^{N}, T_{c}^{m}\right)-W_{i}\left(\left\{Y_{d, i}^{m}\right\}_{i=1}^{N}, T_{d}^{m}\right)\right]
\end{aligned}
$$

In order to verify the properties $(\mathrm{P} 1)$ and $(\mathrm{P} 2)$, we can make use of the regularity properties of $\left\{W_{i}\right\}_{i=1}^{N}$ and $f_{0}$. The elaboration of the standard energy arguments will again be omitted.

6.5 The auxiliary problem (13). This is the essential step in our investigation, as we pass from an incompressible velocity field to a slightly compressible one. The error mechanisms of Chorin's projection scheme have been outlined in section 4, and we can state the following lemma.

Lemma 6.5 Suppose (A1),(AD) and (A3) to be satisfied for the solution of (1),(D). Then, the solution $\left\{u_{e}^{m+1}, p_{e}^{m+1}, T_{e}^{m+1},\left\{Y_{e, i}^{m+1}\right\}_{i=1}^{N}\right\} \in \mathcal{X}$ of scheme (13) satisfies the properties (P1) and (PQ), provided the time-steps $k \leq k_{0}\left(t_{M+1}\right)$ are chosen sufficiently small.

Remark 6.1 Note that the driving forces in the momentum equations in (12) and (13) are identical and that the 'chemical parts' are the same. Thanks to Lemma 6.4, the regularity of the right hand side in (13) enables the application of Lemma 4.1.

6.6 The auxiliary problem (14). This section is devoted to gain further understanding in the effect the projection scheme has on the temperature and the concentration in scheme (4) through (7), or the reformulation (14).

Lemma 6.6 Assume the assumptions (A1),(AD) and (A3) to be valid. Then, the solution $\left\{u_{f}^{m+1}, p_{f}^{m+1}, T_{f}^{m+1},\left\{Y_{f, i}^{m+1}\right\}_{i=1}^{N}\right\} \in \mathcal{X}$ of scheme (14) satisfies the properties (P1) and (PQ), provided the time-steps $k \leq k_{0}\left(t_{M+1}\right)$ are sufficiently small.

Proof. By using the error notation

$$
\begin{aligned}
& e^{m+1}:=u_{e}^{m+1}-u_{f}^{m+1}, \quad \eta^{m+1}:=p_{e}^{m+1}-p_{f}^{m+1}, \\
& \mathcal{T}^{m+1}:=T_{d}^{m+1}-T_{f}^{m+1}, \quad \mathcal{Y}_{i}^{m+1}:=Y_{d, i}^{m+1}-Y_{f, i}^{m+1},
\end{aligned}
$$


we obtain the following system of error equations,

$$
\begin{aligned}
& d_{t} e^{m+1}-\Delta e^{m+1}+\left(P_{\mathbf{J}_{0}} u_{e}^{m} \cdot \nabla\right) e^{m+1}+\left(P_{\mathbf{J}_{0}} e^{m} \cdot \nabla\right) u_{f}^{m+1} \\
& \quad+\nabla \eta^{m}=\left[f_{0}\left(T_{d}^{m}\right)-f_{0}\left(T_{f}^{m}\right)\right] \\
& \begin{array}{l}
\operatorname{div} e^{m+1}-k \Delta \eta^{m+1}=0,\left.\quad \partial_{n} \eta^{m+1}\right|_{\partial \Omega}=0 \\
d_{t} \mathcal{T}^{m+1}-\Delta \mathcal{T}^{m+1}+\left(P_{\mathbf{J}_{0}} u_{d}^{m} \cdot \nabla\right) \mathcal{T}^{m+1}+\left(P_{\mathbf{J}_{0}}\left[e^{m}+\left(u_{d}^{m}-u_{e}^{m}\right)\right] \cdot \nabla\right) T_{d}^{m+1} \\
\quad=-\sum_{i=1}^{N} h_{i}\left[W_{i}\left(\left\{Y_{d, i}^{m}\right\}_{i=1}^{N}, T_{d}^{m+1}\right)-W_{i}\left(\left\{Y_{f, i}^{m}\right\}_{i=1}^{N}, T_{f}^{m+1}\right)\right], \\
d_{t} \mathcal{Y}_{i}^{m+1}-\Delta \mathcal{Y}_{i}^{m+1}+\left(P_{\mathbf{J}_{0}} u_{d}^{m} \cdot \nabla\right) \mathcal{Y}_{i}^{m+1}+\left(P_{\mathbf{J}_{0}}\left[e^{m}+\left(u_{d}^{m}-u_{d}^{e}\right)\right] \cdot \nabla\right) Y_{d, i}^{m+1} \\
\quad=\left[W_{i}\left(\left\{Y_{d, i}^{m+1}\right\}_{i=1}^{N}, T_{d}^{m}\right)-W_{i}\left(\left\{Y_{f, i}^{m+1}\right\}_{i=1}^{N}, T_{f}^{m}\right)\right] .
\end{array}
\end{aligned}
$$

The energy analysis is again evident, and (P2) is satisfied - apart from the first step that involves the treatment of the pressure term:

$$
\begin{aligned}
\frac{1}{2} d_{t}\left\|e^{m+1}\right\|^{2}+\frac{1}{2} k \| d_{t} e^{m+1} & \left\|^{2}+\right\| \nabla e^{m+1}\left\|^{2}+\frac{1}{2} k\right\| \nabla \eta^{m+1} \|^{2} \\
& \leq C\left\{\left\|\mathcal{T}^{m}\right\|^{2}+\left\|e^{m+1}\right\|^{2}\right\}+\frac{1}{2} k^{3}\left\|\nabla d_{t} \eta^{m+1}\right\|^{2} .
\end{aligned}
$$

Because of the following inequality that is a consequence of the second equation in (31),

$$
k^{2}\left\|\nabla d_{t} \eta^{m+1}\right\|^{2} \leq\left\|d_{t} e^{m+1}\right\|^{2},
$$

the second term on the right hand side can be absorbed by the left hand side. - As already mentioned, the remainder of the verification of the approximation property (P2) will be omitted.

As a consequence of the upper error analysis, we can easily obtain a couple of a priori bounds for the solution of (14). The bound

$$
\max _{0 \leq m \leq M}\left\|\nabla p_{f}^{m}\right\|^{2} \leq C
$$

is a consequence of the error bound (32) and the second identity in (31), together with Lemma 6.5, and the following two bounds,

$$
k \sum_{m=0}^{M}\left\{\left\|\Delta u_{f}^{m+1}\right\|^{2}+\left\|d_{t} u_{f}^{m+1}\right\|^{2}\right\} \leq C
$$

then easily follow from the first identity in (14). - Now, we can apply $d_{t}$ to the first equation of (14) and test it with $d_{t} u_{f}^{m+1}$. We can make use of the previous results to finally verify the following inequality,

$$
\left\|d_{t} u_{f}^{M+1}\right\|^{2}+\left\|\Delta u_{f}^{M+1}\right\|^{2}+k \sum_{m=0}^{M}\left\{\left\|\nabla d_{t} u_{f}^{m+1}\right\|^{2}+k\left\|\nabla d_{t} p_{f}^{m+1}\right\|^{2}\right\} \leq C .
$$


This proves the properties (P1) for the velocity field. The easy verification of the corresponding properties for the temperature field and the $N$ concentrations is omitted.

7 Proof of Corollary 2.1 From the analysis of the auxiliary problems (9) through (28) that has been performed in the previous sections, we have striking stability results (given by property $(\mathrm{P} 1))$ and $\ell^{\infty}\left(0, t_{M+1} ; L^{2}(\Omega)\right)$-convergence results (given by property $(\mathrm{P} 2)$ ) at hand for the velocity components, the temperature distribution and the mass fractions. Coming back to the third and fourth error equations in each of the identities (22), (27), (29), $(30)$, and (31), we can make use of these results.

We start with (22), since it is exeptional from the technical point of view: due to (23), we have to test with time weighted functions. For example, if we consider the third equation, testing with $-\tau_{m+1} \Delta \mathcal{T}^{m+1}$ finally leads to

$$
\tau_{M+1}\left\|\nabla \mathcal{T}^{M+1}\right\|^{2}+k \sum_{m=0}^{M} \tau_{m+1}\left\|\Delta \mathcal{T}^{m+1}\right\|^{2} \leq C k^{2}+k \sum_{m=0}^{M}\left\|\nabla \mathcal{T}^{m}\right\|^{2}
$$

The latter inequality is a result of Gronwall's discrete inequality and Remark 5.1. The last term can now be bounded by $C k^{2}$, using (24) (in summed version).

In the second part of this section, we now focus on the remaining error systems, exept from system (22). Since the arguments can be given in the same way for each of the systems, we can confine on illustrating it for the third equation of system (27). If we test this equation with $-\Delta \mathcal{T}^{m+1}$, we obtain after summation over all iteration steps,

$$
\begin{aligned}
& \frac{1}{2}\left\|\nabla \mathcal{T}^{M+1}\right\|^{2}+k \sum_{m=0}^{M}\left\|\Delta \mathcal{T}^{m+1}\right\|^{2} \leq C k \sum_{m=0}^{M} \sum_{i=1}^{N}\left\{\left\|Y_{a, i}^{m+1}-Y_{b, i}^{m+1}\right\|^{2}+\left\|T_{a}^{m+1}-T_{b}^{m+1}\right\|^{2}\right\} \\
& \quad+C k \sum_{m=0}^{M}\left\{\left\|u_{a}^{m+1}\right\|_{1}\left\|\mathcal{T}^{m+1}\right\|_{1}^{1 / 2}\left\|\Delta \mathcal{T}^{m+1}\right\|^{3 / 2}+\left\|e^{m+1}\right\|_{0, q}\left\|\nabla T_{b}^{m+1}\right\|_{0, r}\left\|\Delta \mathcal{T}^{m+1}\right\|\right\},
\end{aligned}
$$

for positive numbers $q, r$ satisfying $\frac{1}{q}+\frac{1}{r}=\frac{1}{2}$. The first sum can be bounded by $C k^{2}$, thanks to Lemma 6.2. In order to deal with the first part of the second sum, we can apply Young's inequality twice to absorb terms on the left hand side of the inequality.

Owing to different Sobolev inequalities for $d=2,3$, we have to deal with the last term in (35) independently for both cases. We start with the case $d=2$. Thanks to the GagliardoNirenberg interpolation inequality (see, [16], e.g.), we obtain for positive values $q$,

$$
\begin{aligned}
& \leq C\left\{k^{2}+k \sum_{m=0}^{M}\left\|\nabla \mathcal{T}^{m+1}\right\|^{2}\right\}+\frac{1}{4} k \sum_{m=0}^{M}\left\|\Delta \mathcal{T}^{m+1}\right\|^{2} \\
& \quad+C k \sum_{m=0}^{M}\left\|e^{m+1}\right\|^{4 / q}\left\|\nabla e^{m+1}\right\|^{2-4 / q}\left\|\nabla T_{b}^{m+1}\right\|_{0, \frac{2 q}{q-2}}^{2}
\end{aligned}
$$




$$
\leq C\left\{k^{2}+k^{4 / q+1-2 / q} \max _{0 \leq m \leq M}\left\|T_{b}^{m+1}\right\|_{2}\right\}+\frac{1}{4} k \sum_{m=0}^{M}\left\|\Delta \mathcal{T}^{m+1}\right\|^{2},
$$

using again Lemma 6.2 (property (P2)). Furthermore, we made use of the embedding inequality $\|\phi\|_{0, q} \leq C\|\phi\|_{1}, \forall 1 \leq q<\infty$. Note, that the constant $C$ in the last inequality in (36) depends on the choice of the value $q$, and goes to infinity as $q \rightarrow 2$. The last term in (36) can be absorbed on the left hand side of (35).

For the three-dimensional case $(d=3)$, we can continue in (35) in the following way,

$$
\begin{gathered}
\leq C\left\{k^{2}+k \sum_{m=0}^{M}\left\|\nabla \mathcal{T}^{m+1}\right\|^{2}\right\}+\frac{1}{4} k \sum_{m=0}^{M}\left\|\Delta \mathcal{T}^{m+1}\right\|^{2} \\
+C k \sum_{m=0}^{M}\left\|e^{m+1}\right\|\left\|\nabla e^{m+1}\right\|\left\|T_{b}^{m+1}\right\|_{2}^{2} \leq C k^{3 / 2}
\end{gathered}
$$

The latter inequality is a consequence of Lemma 6.2 (property (P2)). Corresponding arguments furnish the statement of Corollary 2.1.

Remark 7.1 As it turns out from the latter considerations, suboptimal convergence behavior for the gradients of temperature distribution and mass fraction in (4) through (7) is caused by the nonlinear character of system (1). More precisely, the actual "bottle-neck" can be found in the auxiliary problem (13) that is devoted to the study of the pressure-stabilization feature of the method. In this contents, the gradient of the velocity field can only be approximated of the order $\mathcal{O}\left(k^{1 / 2}\right)$ in the norms $\ell^{\infty}\left(0, t_{M+1} ; \mathbf{H}_{0}^{1}\right)$ or $\ell^{2}\left(0, t_{M+1} ; \mathbf{H}_{0}^{1}\right)$.

8 Conclusion and Outlook. In the present work, we proposed and analyzed the time-splitting scheme (4) through (7) that decouples the computation of approximates in each iteration step. This approach reduces the computational effort in a significant way, by solving equations for each of the quantities $\left\{u^{m+1}, p^{m+1}, T^{m+1},\left\{Y_{i}^{m+1}\right\}_{i=1}^{N}\right\} \in \mathcal{X}$ independently in each iteration step. This scenario is justified by a rigorous error analysis, with optimal convergence results given in Theorem 2.1 and Corollary 2.1. In particular, the scheme is suitable for parallelization strategies, and can be combined with effective finite element discretizations that take into account specific spatial and temporal features of the involved quantities. - A basic ingredient of the algorithm is the projection method of Chorin, for which modest approximation of the pressure iterate in the vicinity of the boundary $\partial \Omega$ is known, owing to the prescription of homogeneous boundary data in (8), which leads to a boundary layer. This topic has been extensively analyzed in [13], where also a new projection-type method has been proposed and investigated that avoids this drawback of Chorin's method, called the Chorin-Uzawa method. In particular, the application of the Chorin-Uzawa projection method - instead of the classical Chorin scheme - in this context leads to error statements that are superior to those given in Corollary 2.1. This is due to an improved error statement for the gradient of the velocity field for the approximation of the Navier-Stokes equations (15), see Theorem 8.2 in [13], which can be successfully applied in 
(37). This finally leads to first order convergence results for the corresponding error quantities given in Corollary 2.1, even in three space dimensions.

Acknowledgment: The research was conducted during my stay in the IMA, University of Minnesota, and has been supported by a DFG-scholarship. I thank the IMA for the hospitality. In particular, I am grateful to Prof. G. Sell for bringing this topic to my mind and many stimulating discussions.

\section{References}

[1] K..J. Bachmann, H.T. Banks, N. Dietz, K. Ito, J.S. Scroggs, H.T. Tran, Modeling and Control of Advanced Chemical Vapor Decomposition Processes, in: Mathematics of Microstructure Evolution (eds.: L.Q. Chen, B. Fultz, J.W. Cahn, et al.), The Minerals, Metals \& Materials Society, 1996, pp. 327-344.

[2] F. Brezzi, M. Fortin, Mixed and Hybrid Finite Element Methods, Springer Series in Computational Mathematics (1991).

[3] A. J. Chorin, Numerical solution of the Navier-Stokes equations, Math. Comp. 22 (1968), pp. 745-762.

[4] A. J. Chorin, On the convergence of discrete approximations of the Navier-Stokes equations, Math. Comp. 23 (1969), pp. 341-353.

[5] V. Girault, P.A. Raviart, Finite Element Methods for Navier-Stokes Equations, Springer, Berlin-Heidelberg, 1986.

[6] J.G. Heywood, R. Rannacher, Finite element approximation of the nonstationary Navier-Stokes Problem. I. Regularity of solutions and second order error estimates for spatial discretization, SIAM J. Numer. Anal. 19 (1982), pp. 275-311.

[7] P. Knobloch, Solvability and finite element discretization of a mathematical model related to Czochralski crystal growth, Ph.D thesis, Preprint MBI-96-5 (Univ. of Magdeburg), 1996.

[8] M. Luskin, R. Rannacher, On the smoothing property of the Galerkin method for parabolic equations, SIAM J. Numer. Anal. 19 (1981), pp. 93-113.

[9] O. Manley, M. Marion, and R. Temam, Equations of combustion in the presence of complex chemistry, Indiana University Mathematics Journal 42 (1993), no. 3, 941-967.

[10] M. Marion and R. Temam, Some remarks on turbulent combustion from the attractor point of view, Math. Modelling in Combustion and Related Topics, vol. 35, Martinus Nyhoff, Dordrecht, 1988, pp. 155-172.

[11] Oran, E.S., Boris, J.P., Numerical Simulation of Reactive Flows, Elsevier Science Publ. Inc.: New York, 1987. 
[12] D.A. Norman, A Model for chemically reacting fluid flows: weak solutions and global attractors, IMA Preprint Series no. 1487, June 1997.

[13] A. Prohl, Projection and quasi-compressibility methods for solving the incompressible Navier-Stokes equations, Teubner, 1997.

[14] A.G. Salinger, J.N. Shadid, H.K. Moffat, S.A. Hutchinson, G.L. Hennigan, K.D. Devine, Parallel Reacting Flow Calculations for Chemical Vapor Deposition Reactor Design, in: Proceedings of the International Conference on Computational Engineering Science, San Jose, Costa Rica, May 4-9, 1997.

[15] W. Seifert, Numerische Behandlung freier Ränder beim Kristallziehen, Ph.D thesis, TU Munich, 1996.

[16] G.R. Sell, Y. You, Dynamics of evolutionary equations, Lecture Notes, 1996.

[17] R. Temam, Navier-Stokes Equations, 3d rev. ed., North Holland, Amsterdam, 1984.

[18] F.A. Williams, Combustion theory: the fundamental theory of chemically reacting flow systems, 2nd ed., Benjamin/Cummings Pub. Co., Menlo Park, Calif., 1985. 